I now had recourse to the ergot of rye, two scruples of which $I$ instantly gave for a dose in half a tumbler of hot water. In about ten minutes a copions emesis took place, which was followed by a warm glow, and a profuse moisture over the entire surface. The same quantity was repeated, which almost immediately caused a strong pain, and contracted from the fundus towards the neck of the uterus, with forcible bearing-down, so as, with a jerk, completely to empty itself of its contents. Recovery proved rapid and complete.

In conclusion, I feel confident, that in the hands of the judicious obstetrician, in any of the three stages of labour, the ergot of rye is a most admirable adjuvant to the operations of Nature; that in cases of retention of the placenta, where uterine action is suspended altogether, or tardy, whether there be hæmorrhage or not, it may be safely administered, and generally with complete success; and that even where extraction may be deemed expedient, it proves a most worthy assistant in inducing uterine action in this very important phase of labour. The objection, that a corresponding relaxa. tion of the uterus is liable to follow its use, is, in my opinion, an exaggeration-in fact, my experience proves the reverse, and if it should occur; a repetition of the dose is generally effectual.

London, 1848.

\section{ON THE PATHOLOGICAT TREATMENT OF HOOPING-COUGH.}

Bx J. PIDDUCK, M.D., \&c., London.

THE importance of having some clearly-defined principle as a guide in the treatment of every disease is so evident, that no arguments are required in its proof. The want of such a principle is particularly felt in the treatment of hooping-cough. Of all the diseases which come under the cognizance of the physician, there is scarcely one that more frequently baffles his skill, or is less under the control of medicine, than hoopingcough. After subduing inflammatory and febrile symptoms at the commencement of the complaint, the practice, for the most part, ceases to be rational, and becomes empirical. Emetics, purgatives, diaphoretics, antispasmodics, counterirritants, specifics of various kinds, and lastly, tonics, are tried in succession; and these failing, change of air is recommended as the dernier ressort.

Pathological anatomy has supplied the principle which leads to a rational practice in this disease. It has demon. strated the existence of a congested state of the vessels at the origin of the pneumogastric and other respiratory nerves, and a more copious effusion of serum around the medulla oblongata than in death from other causes, except those involving diseases of the lungs and heart.

It was the discovery of this state of the vessels at the origin of these nerves, by the late Dr. Sanders, of Edinburgh, which led him to a rational and successful practice in hooping-cough. It consists in applying leeches directly over the junction of the occiput and the atlas vertebra, for the purpose of relieving the congested state of those vessels, followed by a blister between the shoulders, to promote their contraction. The rubefacient effect of the blister is sufficient to answer this indication, and therefore, in delicate children, a mustard-poultice is preferable to a blister. The rule to be observed is, to apply one leech for each year of the child's age, from one to six; and immediately after the leeches, the small blister or sinapism; and to repeat the leeches and rubefacient on the third or fourth day, if necessary.

The first application usually succeeds in arresting the violent paroxysms of the cough; sometimes a second, but very rarely a third application is required to put an end to the paroxysms.

During the period of nearly thirty years that $I$ have pursued this rational practice, I cannot recollect a single instance of failure in uncomplicated cases of hooping-congh. It is unnecessary to point out to the physician of judgment and experience, especially to those who have studied the intimate relation which subsists between innervation and the diseases of the respiratory and circulatory systems, the importance of attending to the pathological condition of that most directly influential part of the nervous system-viz., the origin of the pneumogastric and respiratory nerves. In the great majority of these cases, heat, redness, and tenderness on pressure, indicate the state of the part subjacent.

This mode of treatment applies strictly to the uncomplicated cases of hooping-cough. The several complications require their separate and appropriate modes of treatment. Leeching the upper part of the spine, and blistering between the shoulders, by arresting the violence of the cough, speedily remove the congested and inflammatory states of brain which the hooping-cough frequently occasions. The catarrhal complication requires the exhibition of the wine of colchicum, combined with an alkali, after clearing the alimentary canal. The bronchitic and pneumonic complications require the administration of the potassio-tartrate of antimony with nitrate of potass; and the biliary complication, of mercury and rhubarb, with saline aperients.

If none of these complications are present, or after they are. removed, if the child is delicate, tonics (and pure air is perhaps the best) complete the cure.

The symptomatic proof of the congested state of the vessels. surrounding the origin of the pneumo-gastric nerves, is the. vomiting, which frequently terminates the paroxysm-a symptom which almost invariably attends an injury done to this part of the cerebro-spinal system by the contre-coup, from a fall, or heavy blow on the head. It is this symptom which has led to the irrational practice of giving emetics; and because. the food is rejected in an acid state-that is, during the first. stage of digestion, when the contents of the stomach are invariably acid, to the still more irrational alkaline treatment. It is, however, true, that an emetic may be useful to clear thestomach and duodenum from morbid secretions, and the bronchi from mucus, after the application of the leeches and blister.

Montague-street, Bloomsbury, 1849.

\section{ON CERTAIN DISPUTED OBSTETRIC INVEN- TIONS, AND CERTAIN MODERN SURGICAL.} IMPROVEMENTS.

\section{By JAMES ARNOTT, M.D., \&c., Brighton.}

Dr. SLrman and Dr. Cattell have each, (as appears, ) and quite independently, lately suggested the expedient, in uterine hæmorrhage, of introducing a vladder into the uterus, and afterwards injecting or distending it with cold water; and Dr. Cattell complains of his precedence in the invention-a precedence of a twelvemonth-having been neglected. It may lessen his annoyance to be informed, that if he refers to an article on uterine hremorrhage, in a very recent number of The Lancer, (that, I think, of the 22nd of last December, ) he will find an account of a similar suggestion which was made by myself so long ago as 1845 , and published in the appendix to iny Essay "On the Present State of Therapeutical Inquiry." There is this important difference, however, between what I have suggested and the proposal of Dr. Slyman, (and it is this difference which induces me now to advert to the subject, in order that the suggestion may not lead to injurious practice,) that the pressure in the former is made by the weight of water in an open vertical tube; whereas, in the other plan, it is made by the force of the operator's hand, and after the water has been injected, its escape is prevented by a stop-cock. To begin the injection as $I$ have recommended, a greater force than that of a moderate column of water may be necessary; but if the tube were closed, as suggested by Drs. Slyman and Cattell, the uterus would be prevented contracting, as it could not expel the water from the bladder.

Ludicrous as this claim from two different parties may be, made in the very journal which, only a few weeks before, contained a description of this supposed invention, it is not more singular than some cqually recent claims which have been preferred in the same jourual by Messrs. Critchett and Chapman for improvements in the treatment of ulcers. Neither of these gentlemen asserts that the great principle of support from pressure was unknown before his publications; but they found their claims on the mode of making this pressure, and of combining the above principle with that of subduing the accompanying inflammation. The only difference, however, in their prescribed mode of treatment from what is now generally in use, and has been in use for the last forty years, is some trifling difference in the mode of applying straps or bandages. Now, as I have for several years past been using and publicly recommending a perfect mode of applying and regulating pressure, and a perfect mode of regulating the temperature of the part at the same time, (which is by far the best plan of reducing any accompanying inflammatory action,) I cannot omit this opportunity of expressing my surprise at this more recent recommendation of methods of fulfilling the two indications alluded to, so obviously imperfect and defective. If it be objected that the treatment by fluid pressure, and the "current apparatus," confines a patient to his couch; the 